\title{
Research on dynamic error caused by three coordinate measuring machine body deformation
}

\author{
Qu Ligang ${ }^{1, a}$, Liang $\mathrm{Yu}^{1, \mathrm{~b}^{*}}$ and Su Changqing ${ }^{1, \mathrm{c}}$ \\ ${ }^{1}$ Key Laboratory Fundamental Science for National Deference of Aeronautical Digital Manufacturing \\ Process, Shenyang Aerospace University, Liaoning, Shenyang, 110000, China \\ a 18804072122@163.com, ${ }^{\text {b3 } 358053621 @ q q . c o m, ~ c n e u s u c q @ 163 . c o m ~}$
}

\begin{abstract}
Keywords: Three coordinate measuring, Inertia force, Body deformation, Dynamic error Abstract. In high precision three coordinates measuring machine in the process of measurement, dynamic error analysis has been improved measuring machine precision and speed is an important subject. Three coordinate measuring machine structure in the movement of the dynamic deformation of the theoretical points. Due to the servo motor driving force and moving inertia force components will lead to the overall bridge, carriage, upright post and a $\mathrm{Z}$ shaft and other components deflect. And through the corresponding theoretical calculation, it is pointed out that the bending deformation of the main structure of the air bearing guide of the components is one of the main causes of the dynamic error. By doing dynamic error experiments, the dynamic errors of CMM body deformation was measured to verify the impact of the body deformation and probe position accuracy, optimize the design of error correction and institutions provide the basis.
\end{abstract}

\section{Introduction}

From the $50 \mathrm{~s}$ of last century, the coordinate measuring machine (CMM) produced up to now a variety of three coordinate measuring machine in industry widely used, measuring equipment has clearly become modern manufacturing industry is unable to substitute a constitute the main part of $[1,2]$. In the past nearly 100 years of history, manufacturing using coordinate measuring machine after more than a qualitative leap. With the continuous development of industry, the three coordinate measuring machine demand is higher and higher, the high precision and high speed measuring machine is more and more popular, each manufacturer are introduced under the condition of guarantee the accuracy of the measuring machine, the most widely used for mobile bridge three coordinate measuring machine. The speed of the measuring machine is more and more fast, and the accuracy is more difficult. How to improve the speed is an important subject in the condition of the guarantee accuracy.

Now air flotation guide rail is widely used in the design of the measuring machine. In the process of high speed measurement, measuring machine parts acceleration will lead to stress the role, due to the stiffness of aerostatic guide way and the institution itself enough, measuring machine makes the moving parts and air guide structure itself to bending and deflection, connecting the moving parts of air bearing slider gap size will change, resulting in a grating reading and measuring lines do not coincide, actual measurement value and grating reading is different, resulting in the dynamic error $[3,4]$. In general, the greater the acceleration, the greater the inertia force, the greater the interaction force between the various moving parts, the greater the deflection and bending deformation errors occur. In this paper, the generation mechanism of the body's dynamic deformation (including the dynamic deformation and dynamic deformation) is studied, to set up the body structure dynamic deformation and displacement error transformation model, and verify the difference of the spatial position, the displacement error caused by the body's dynamic deformation is also different, which is of great significance to improve the work efficiency and structural optimization.

\section{Dynamic deformation analysis}

The main research of this paper is the most widely used in the factory bridge CMM, such as shown in Figure 1, at the bottom of the fixed table, move the sliding frame can be on the bridge, to move along the $\mathrm{Y}$ axis, measuring head system and the $\mathrm{Z}$ axis is fixed on the sliding frame along the $\mathrm{Z}$ axis can be 
mobile, the bridge can be to move along the $\mathrm{X}$ direction, $\mathrm{x}, \mathrm{y}, \mathrm{z}$ direction measurement, reciprocally moving parts with air cushion support each other, working table and the beam is generally granite materials and other materials for aluminum alloy materials.

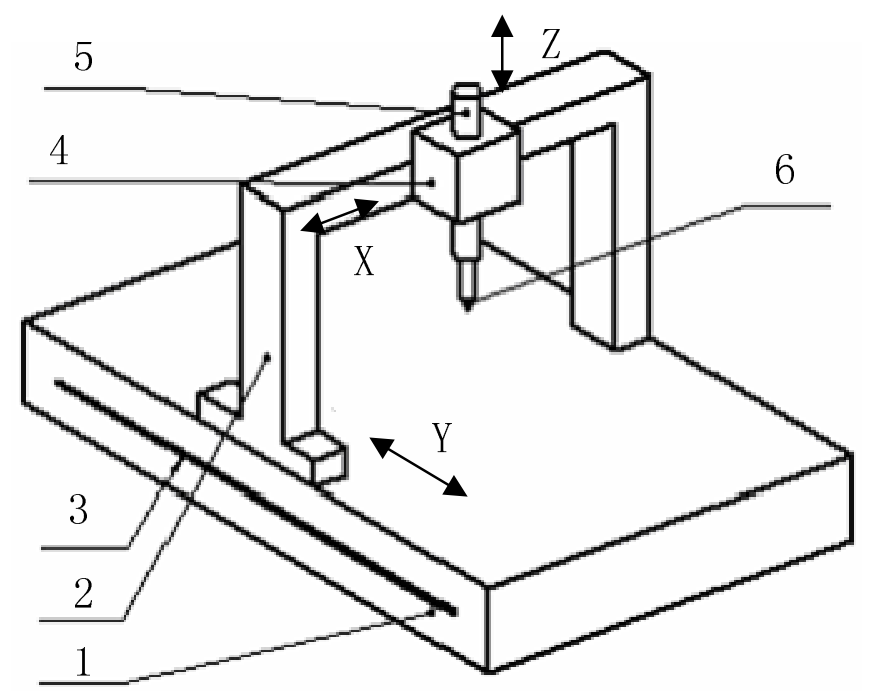

1. Workbench 2.Bridge 3.Measuring scale 4.Carriage 5.Z axis 6.Measuring head

Fig. 1 Schematic diagram of the three coordinate measuring machine

In the three coordinate measuring machine in the process of rapid measurement, due to the effect of inertia force, when the probe contact measurement point is still in a state of acceleration. When measuring head along the $\mathrm{Y}, \mathrm{Z}$ three directions accelerated moves, driving force and inertial force exerting on the measuring machine mechanical structure, lead to the pillar, carriage moving parts deformation .Each part is connected with the air cushion support. When the gap distance between the air floating guide rail is changed, leading to the deviation of the measuring head position. When measuring head moving along the $\mathrm{X}$ direction, When measuring head moving along the $\mathrm{X}$ direction, because the measuring line do not overlap with grating, there is a big abbe error of measurement standard is different from the actual grating reading, it also produce a larger dynamic error. [5,6].In the $\mathrm{X}, \mathrm{Y}, \mathrm{Z}$ three directions, three coordinate measuring machine all moving parts under the effect of driving force and inertial force caused by the probe offset error is shown in figure 2 .

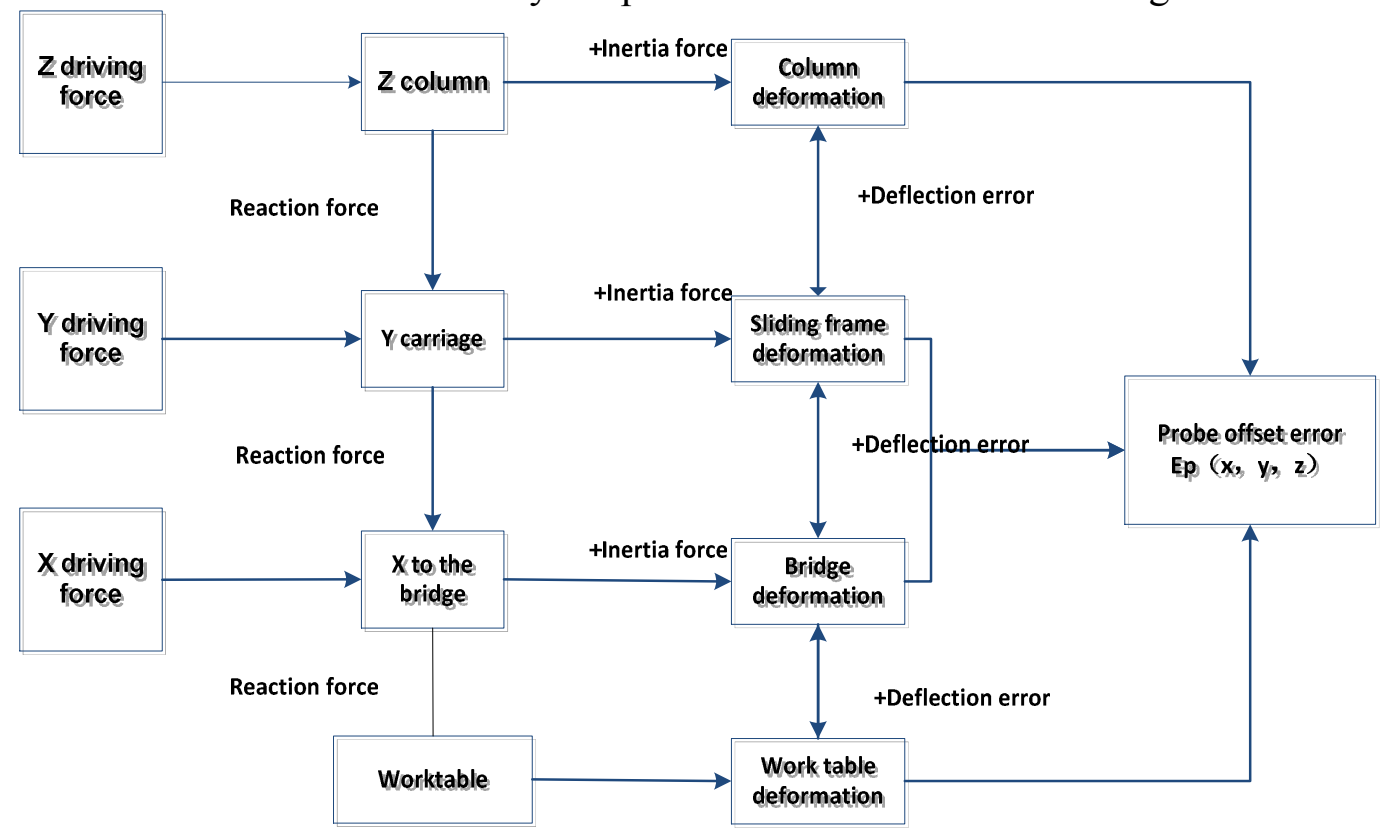

Fig. 2 Mechanism of dynamic deformation 
Because the mobile bridge $\mathrm{CMM}$ is unilateral drive measuring machine, and the dynamic deflection error relative to the displacement error is larger, so we should mainly research the error caused by deflection of air flotation guide in motion. When the $\mathrm{Z}$ axis and carriage move along the $\mathrm{Y}$ direction, due to the quality of the moving parts is small, $\mathrm{Z}$ axis of inertia force on the measurement accuracy has little effect, as a result of the dynamic error is relatively small. So this paper mainly discusses the error caused by dynamic deflection in the $\mathrm{X}$ direction.

\section{Dynamic deflection error of measuring machine}

When measuring machine along the $X$ direction moving at a high speed, driving force and the inertia force will lead to an overall bridge, carriage, column and the $\mathrm{Z}$ axis around the $\mathrm{Y}$ direction and $\mathrm{Z}$ direction deflect. Due to the stiffness of the structure, the mobile bridge itself will deform and bend the deflection error . The movement of the bridge as a whole, the following is $\mathrm{X}$ direction, the moving parts of the deflection error. As shown in the Table 1.

Table 1.Moving part deflection error

\begin{tabular}{|c|c|}
\hline $\begin{array}{l}\text { The deflection angle error of moving bridge around } \mathrm{Z} \\
\qquad \text { axis }\end{array}$ & $\varepsilon_{Z}(X)_{b}$ \\
\hline $\begin{array}{l}\text { The pitching angle error of moving bridge around } \mathrm{Z} \\
\text { axis }\end{array}$ & $\varepsilon_{y}(x) b$ \\
\hline $\begin{array}{l}\text { The deflection angle error of right pillar around the } \mathrm{Z} \\
\text { axis }\end{array}$ & $\varepsilon_{z}(x)_{c}$ \\
\hline $\begin{array}{c}\text { The pitching angle error of right pillar around the } \mathrm{Z} \\
\text { axis }\end{array}$ & $\varepsilon_{y}(x)_{c}$ \\
\hline $\begin{array}{l}\text { The deflection angle error caused by moving bridge } \\
\text { torsion }\end{array}$ & $\varepsilon_{z}(x) t$ \\
\hline $\begin{array}{l}\text { The pitching angle error caused by moving bridge } \\
\text { torsion }\end{array}$ & $\varepsilon_{y}(X)_{t}$ \\
\hline $\begin{array}{l}\text { The deflection angle error of moving bridge around } \\
\qquad \mathrm{Z} \text { axis }\end{array}$ & $\varepsilon_{z}(x)_{p}$ \\
\hline $\begin{array}{l}\text { The pitch angle error of the Y slider and the main } \\
\text { shaft around the Y axis }\end{array}$ & $\varepsilon_{y}(x)_{p}$ \\
\hline
\end{tabular}

When the whole carriage moves along the $\mathrm{X}$ direction, the deflection errors of the $\mathrm{Z}$ and $\mathrm{Y}$ axes are shown in Fig 3, Fig 4.

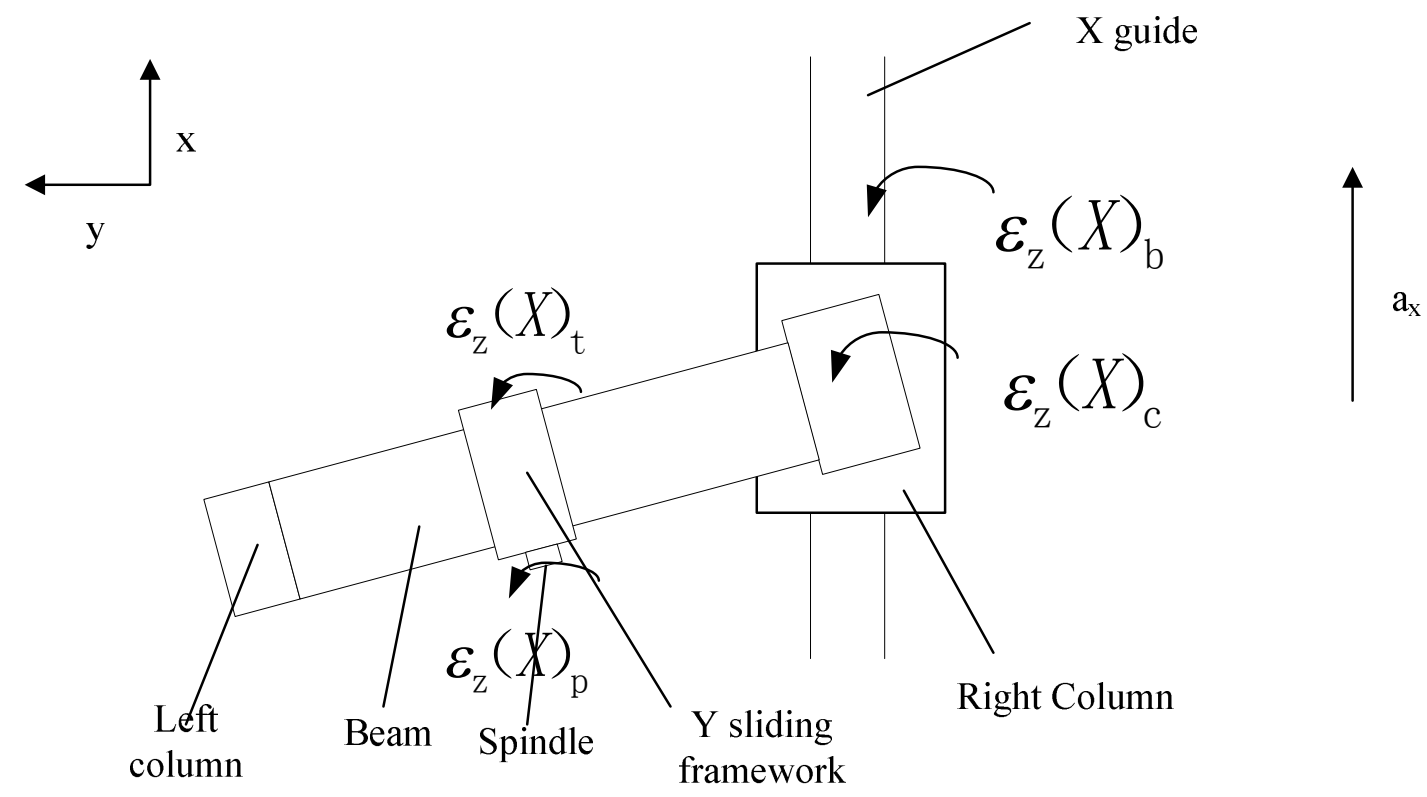

Fig. 3 Deflection error of $Z$ axis 


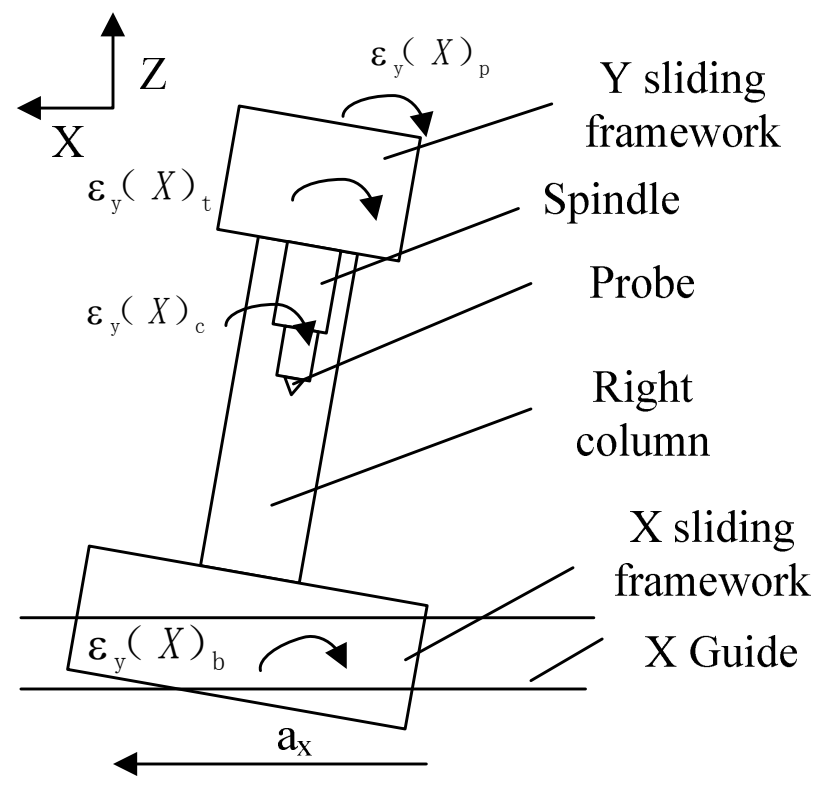

Fig.4 Deflection error of $Y$ axis

\section{The relationship between deviation error and displacement error}

First, we should put the whole moving bridge as a whole to consider, along the $\mathrm{X}$ direction, in the role of inertia force, moving bridge around the $\mathrm{Z}$ axis of the deflection and bending, as shown in Fig 5 shows.

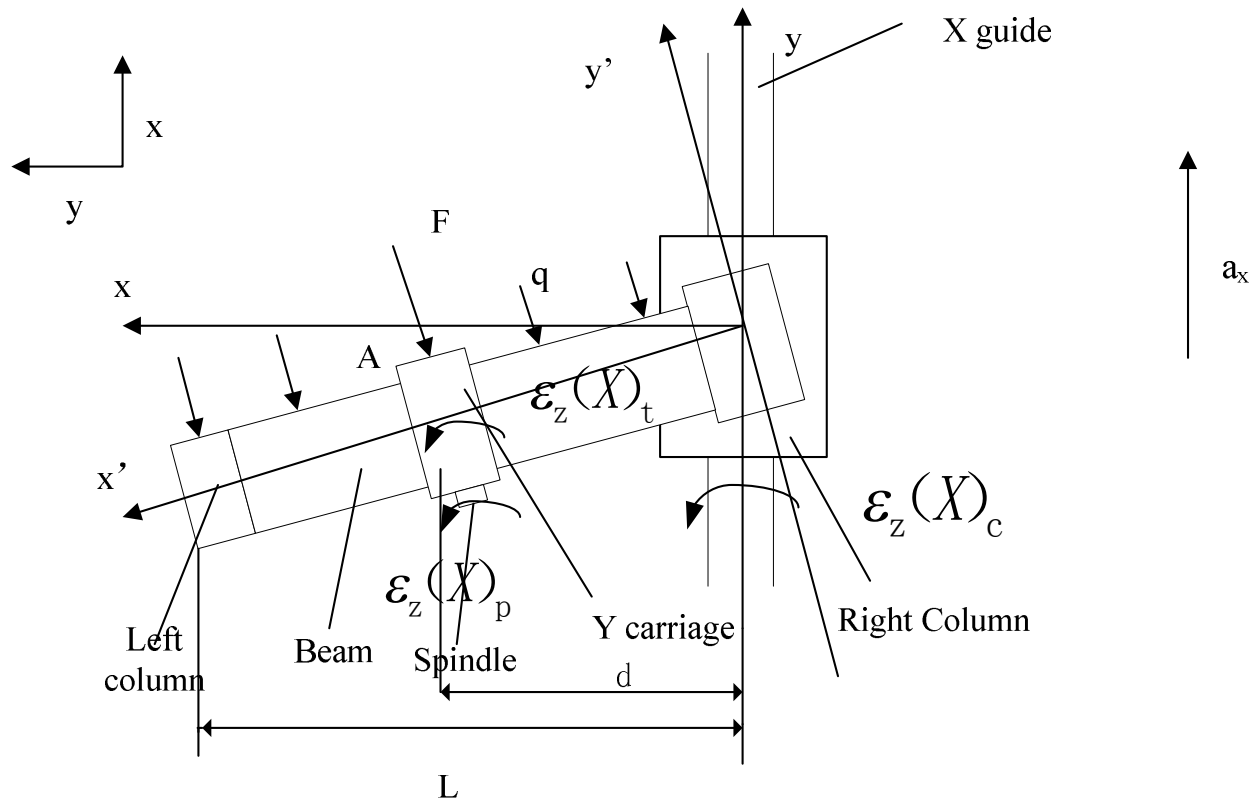

Fig.5 Cantilever beam structure model

As shown in Fig.5, the establishment of a coordinate axis is the same as the direction of the machine itself. Assuming that the total length of the beam is $\mathrm{Y}$, the distance between the L moving carriage and the guide rail is $\mathrm{d}$. Y sliding frame is affected by the inertia force of $\mathrm{F}$, and the distribution of the beam is $\mathrm{q}$. When moving the overall bridge along the $\mathrm{X}$ direction, right column deflection occurred error is $\varepsilon_{Z}(X)_{a}$, Angle $X$ rail system caused by the air guide around the $Z$ axis is $\varepsilon_{z}(x) b$,right angle caused by the torsion pillar pillar of moving bridge right around the $\mathrm{Z}$ axis is $\varepsilon_{\mathrm{z}}(\mathrm{x})_{\mathrm{c}}$.From this we can know $\varepsilon_{\mathrm{z}}(\mathrm{x})_{\mathrm{a}}=\varepsilon_{\mathrm{z}}(\mathrm{x})_{\mathrm{b}}+\varepsilon_{\mathrm{z}}(\mathrm{x})_{\mathrm{c}}$, When the right column angle error occurred, the coordinate axis is changed from oxy to $x$ ' $y$ ' $o$ '. Through the calculation formula of 
engineering mechanics, in the case of different inertia force, the deflection and deflection are calculated as follows. Y slider to bring the focus of the inertial force generating the deflection angle $\theta_{A 1}=\frac{F d^{2}}{2 E I}$, deflection is $f_{1}=-\frac{F d^{3}}{3 E I}$. The inertial force of uniform distribution on the beam $\mathrm{q}$ generating deflection angle $\theta_{A 2}=\frac{q d}{6 E I}\left(d^{2}+3 l^{2}-3 d l\right)$, deflection is $f_{2}=-\frac{q d^{2}}{24 E I}\left(d^{2}+6 l^{2}-4 l d\right)$.Left column inertia concentrated stress $\mathrm{F}_{1}$ mainly generates deflection angle $\theta_{A 3}=\frac{F_{1} d}{2 E I}(2 l-d)$, deflection is $f_{3}=-\frac{F_{1} d^{2}}{24 E I}(3 l-d)$. E is the young's modulus, $\mathrm{I}$ is the inertia moment of the $\mathrm{Z}$ axis. In summary,

$$
\begin{aligned}
& \text { A point deflection angle: } \theta_{A}=\sum_{i} \theta_{A i}=\frac{d}{E I}\left[\frac{F d}{2}+\frac{q}{6}\left(d^{2}+3 l^{2}-3 l d\right)+\frac{F_{1}}{2}(2 l-d)\right] \\
& \text { Deflection: } f=\sum_{i} f_{i}=-\frac{d^{2}}{E I}\left[\frac{F d}{3}+\frac{q}{24}\left(d^{2}+6 l^{2}-4 l d\right)+\frac{F_{1}}{6}(3 l-d)\right]
\end{aligned}
$$

When the whole bridge movement in the direction of $\mathrm{X}$, assume that the total deflection of the $\mathrm{Y}$ slider is $\varepsilon_{z}{ }^{\prime}(x)_{t}, \varepsilon_{z}{ }^{\prime}(x)_{t}=\theta_{A}+\varepsilon_{z}(x)_{a}$. So A point deflection is $\theta_{A}=\varepsilon_{z}{ }^{\prime}(x)_{t}-\varepsilon_{z}(x)_{a}$, Through 4.1, we can get

$$
E I=\frac{d\left[3 F d+q\left(d^{2}+3 l^{2}-3 l d\right)+3 F_{1}(2 l-d)\right]}{6\left[\varepsilon_{z}{ }^{\prime}(x)_{t}-\varepsilon_{z}(x)_{a}\right]} .
$$

Bring 4.3 into the 4.2 formula, we can be concluded that the total deviation error of the A points on the Y slider is

$$
\delta_{x}(x)_{1}=-\frac{2}{3}\left(\varepsilon_{z}^{\prime}(x)_{t}-\varepsilon_{z}(x)_{a}\right) d \frac{\left.F d+q\left(d^{2} / 8+3 l^{2} / 4-l d / 2\right)+F_{1}(3 l-d) / 2\right)}{F d+q\left(d^{2} / 3+l^{2}-l d\right)+F_{1}(2 l-d)} .
$$

Hypothesis $K(d / l)=\frac{F d+q\left(d^{2} / 8+3 l^{2} / 4-l d / 2\right)+F_{1}(3 l-d) / 2}{F d+q\left(d^{2} / 3+l^{2}-l d\right)+F_{1}(2 l-d)}$, the 4.4 formula can be

simplified to

$$
\delta_{x}(x)_{1}=-\frac{2}{3}\left(\varepsilon_{z}^{\prime}(x)_{t}-\varepsilon_{z}(x)_{a}\right) d K(d / l) .
$$

If the calculation includes the offset of the moving carriage relative to the air flotation guide and bending deformation of whole slide inertia force under the action of the frame itself. The formula can be got through the rotation of the coordinates.

$$
\delta_{x}(x)_{1}=-d \sin \left(\varepsilon_{z}(x)_{a}\right)-\frac{2}{3}\left(\varepsilon_{z}^{\prime}(x)_{t}-\varepsilon_{z}(x)_{a}\right) d K(d / l) \cos \left(\varepsilon_{z}(x)_{a}\right) .
$$

Because $\varepsilon_{z}(x)_{a}$ is very small, 4.6 can be simplified as

$$
\delta_{x}(x)_{1}=-d \varepsilon_{z}(x)_{a}-\frac{2}{3}\left(\varepsilon_{z}^{\prime}(x)_{t}-\varepsilon_{z}(x)_{a}\right) d K(d / l) .
$$

The main factors of the deformation of the body are discussed, which lead to the dynamic error of the probe. Including $\mathrm{Y}$ sliding around $\mathrm{Z}$ axis angle $\theta_{A}$ caused by the deformation of the beam, $\mathrm{X}$ guide around the $\mathrm{Z}$ axis angle $\varepsilon_{z}(x)_{b}$ caused by air guide rail system and right angle of torsion $\varepsilon_{Z}(x)_{c}$ caused by the right pillar.

Because the moving bridge pillar and the $\mathrm{Z}$ axis of the anti-deformation ability is strong, moving bridge and spindle around $\mathrm{Y}$ axis deflection $\varepsilon_{y}(x)_{b}$ and $\varepsilon_{y}(x)_{p}$ on the measuring head of the displacement error effects can be adopted $\varepsilon_{y}(x)_{b}$ and $\varepsilon_{y}(x)_{p}$ corresponding Abbe arm length to determine. Effect of displacement error of measuring head deflection and the axis of bridge can be determined by the corresponding Abbe arm length. Assuming the three coordinate measuring 
machine movement of the bridge right pillar height is $\mathrm{h} 1, \mathrm{Z}$ axis extending the length of the $\mathrm{h} 2$. Its displacement error

$$
\delta_{x}(x)_{2}=\varepsilon(x)_{b}\left(h_{1}-h_{2}\right)-\left(\varepsilon_{y}(x) c+\varepsilon_{y}(x) t+\varepsilon_{y}(x)_{p}\right) h_{2} .
$$

So the total displacement error is

$$
\begin{aligned}
& \delta_{x}(x)=\delta_{x}(x)_{1}+\delta_{x}(x)_{2} \\
& =-d \varepsilon_{z}(x)_{a}-\frac{2}{3}\left(\varepsilon_{z}{ }^{\prime}(x)_{t}-\varepsilon_{z}(x)_{a}\right) d K(d, l) \\
& +\varepsilon_{y}(x)_{b} h_{1}-\left(\varepsilon_{y}(x)_{b}+\varepsilon_{y}(x)_{c}+\varepsilon_{y}(x)_{t}+\varepsilon_{y}(x)_{p}\right) h_{2}
\end{aligned}
$$

From the above formula, in the process of moving parts, the structure can produce a large dynamic deformation due to the interaction of driving force and inertia force. It can be seen from the above formula, in the process of moving parts move, due to the driving force and inertia force under the joint action of structures larger dynamic deformation, mainly reflected in all the moving parts on the move around the $\mathrm{X}, \mathrm{Y}, \mathrm{Z}$ axis direction occurs deviation. These dynamic deformation errors can be transmitted to the probe head with the linear dynamic error of the three axis, and the displacement error is generated. According to the formula of 4.9 shows, these body dynamic deformation error not only with the measurement of velocity and acceleration are directly related, but also with the probe relative position change and change, and the relative position of the probe related. The influence of the deformation of the body on the dynamic error of the measuring head is discussed, which provides the theoretical basis for the error correction and mechanism optimization design.

\section{Experimental results of dynamic error caused by structural deformation of measuring machine}

In order to verify the influence of the structural deformation of the measuring machine on the dynamic error, the following experiments are done. Experiments using HP5529A type dual frequency

laser interferometer which measurement accuracy can reach $0.05 \mu \mathrm{m}$, high precision of angle measurement, straightness, vertical experimental data can meet the test requirements. Experimental schematic diagram is shown in Fig 6.

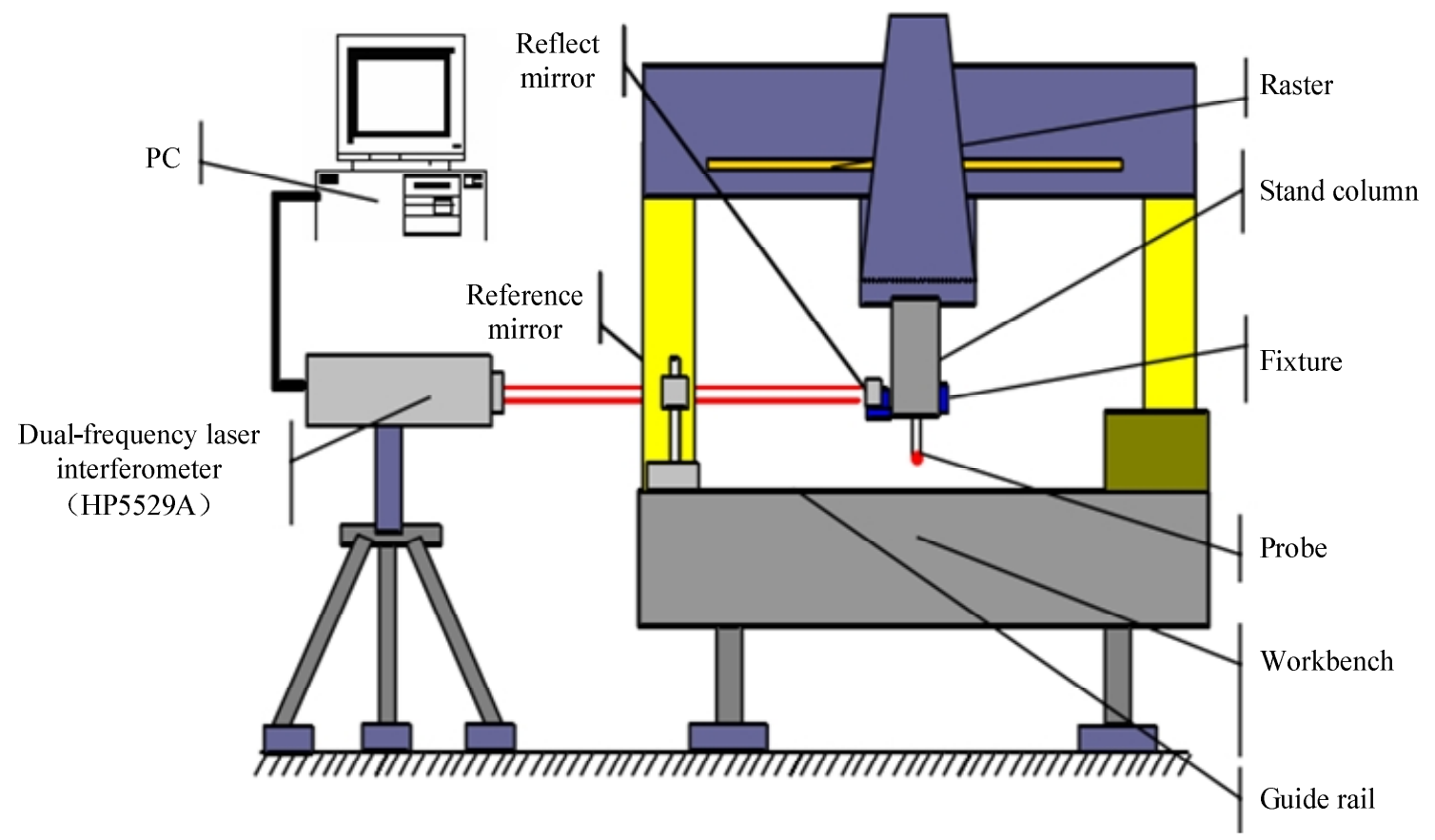

Fig. 6 Experimental schematic diagram

In the measurement error, measuring machines each shaft zero, $\mathrm{Y}$ and $\mathrm{Z}$ axis to keep still, the whole bridge move only in the $\mathrm{X}$-axis direction. Set up the measuring machine bridge $\mathrm{X}$ direction 
movement speed is $40 \mathrm{~mm} / \mathrm{s}$. Based on grating measurement value, the use of HP5529A double-frequency laser interferometer to measure the displacement and displacement of $\mathrm{X}$ axis motion grating detection in real time, then every $20 \mathrm{~mm}$ record error, repeated measures $2-3$ times, the average value of the record, in order to determine when the $\mathrm{X}$ axis movement, $\mathrm{X}$ direction of the angle deviation and displacement deviation. The $\mathrm{X}$ axis displacement error and angle error curve as shown in Fig 7 and Fig 8.

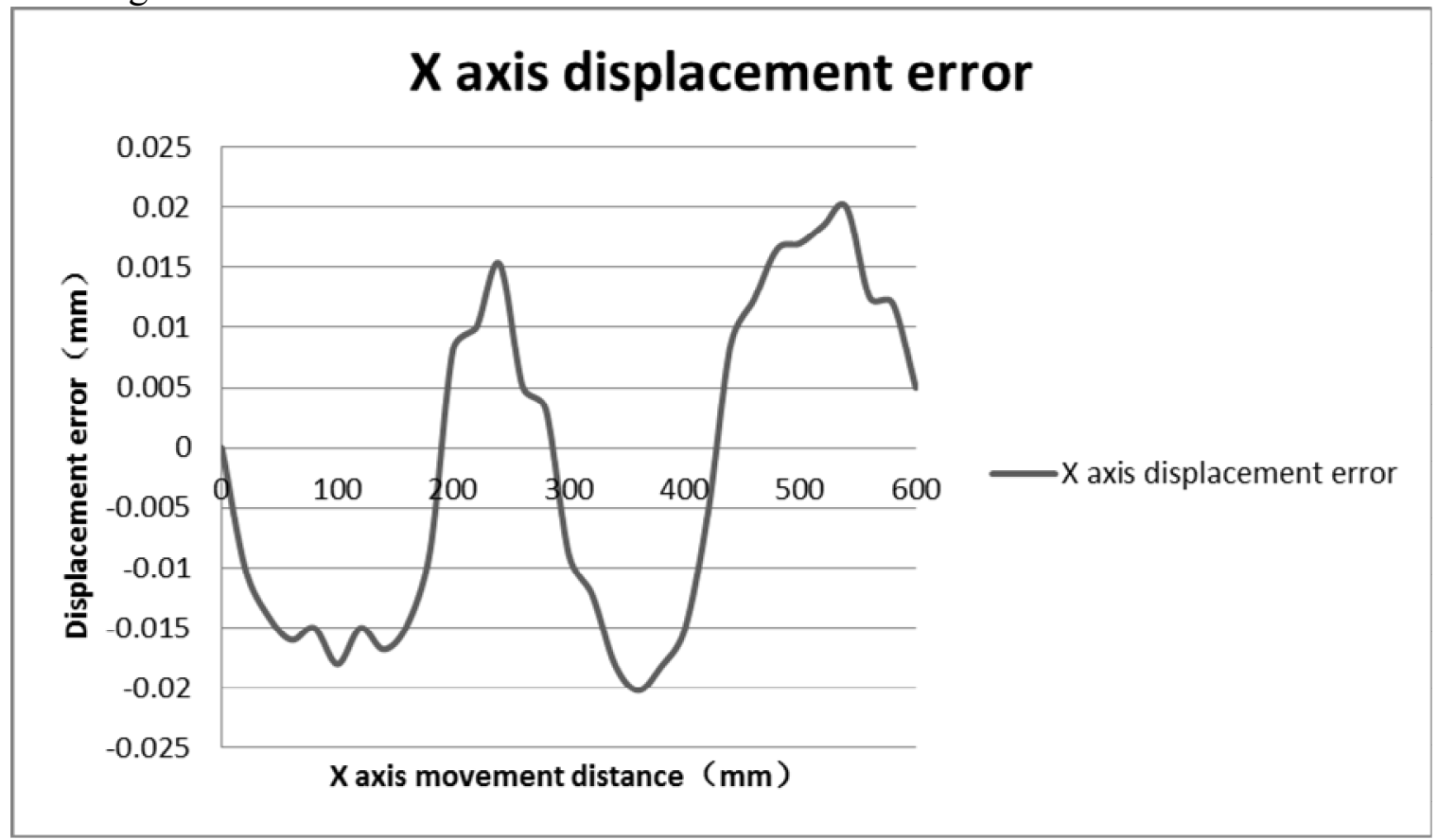

Fig.7 $\mathrm{X}$ axis displacement error curve

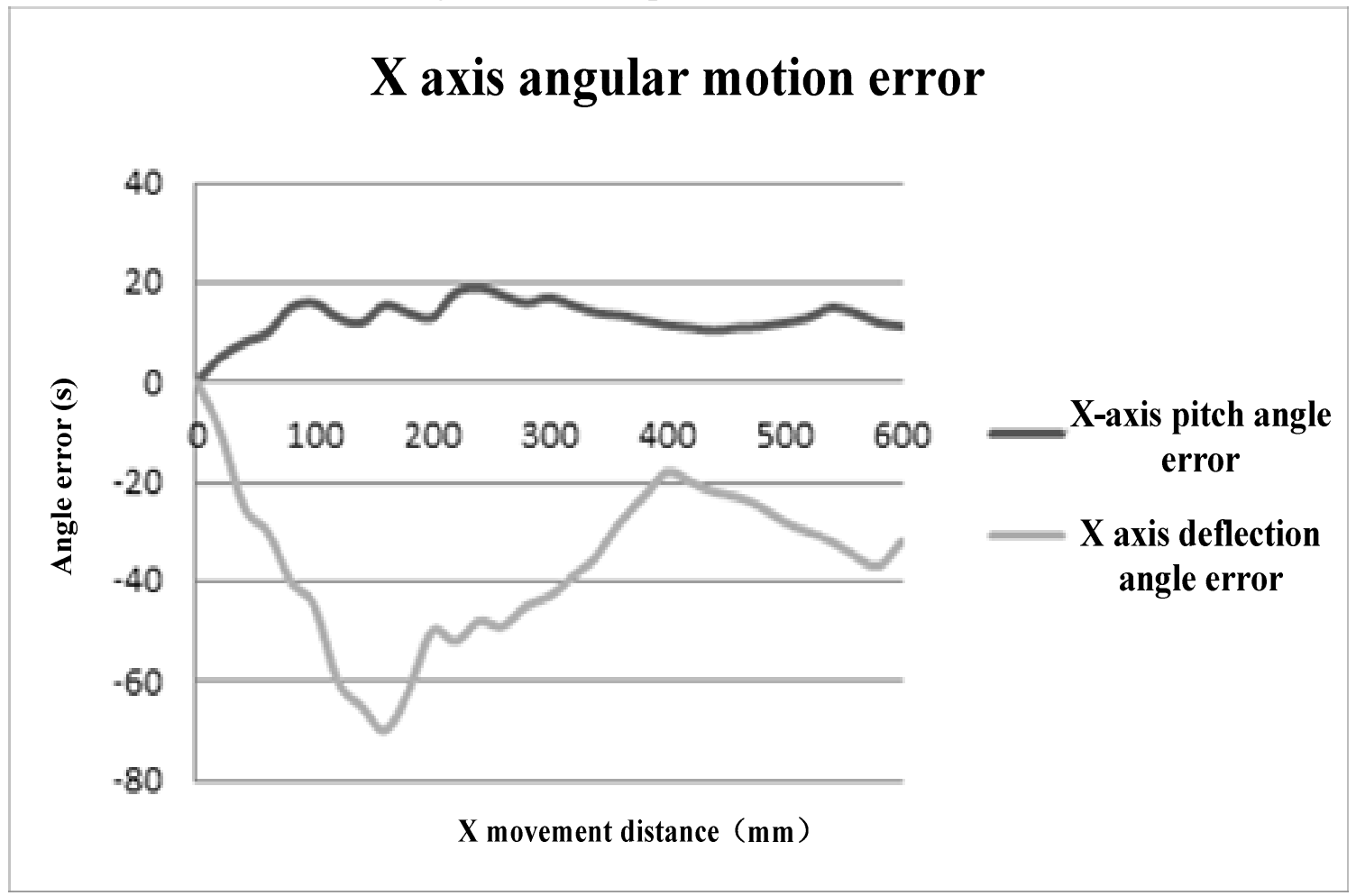

Figure $8 \mathrm{X}$ axis angle deviation curve

Through the above experimental data, we can know all moving parts in the process of moving $\mathrm{x}$, measuring machine parts deformation and torsion effect on the measurement accuracy is bigger, one of the main factors of dynamic error. 


\section{Conclusions}

In this paper, the main error sources of the three coordinate measuring machine are caused by the deflection of the individual components and the bending deformation of the moving parts. Analysis when measuring machine in the $\mathrm{X}$ direction, the impact on the accuracy of measurement of dynamic deformation error has carried on the theoretical analysis and calculation and find out the relationship between the deflection Angle error and displacement error. In theoretical calculation that the body dynamic deformation error not only with the measurement of velocity and acceleration is directly related, but also and the measuring head relative position is directly related to, and dynamic deformation verified by experiment is one of the main factors influencing the measurement precision, better providing theoretical basis for error compensation and correction.

\section{References}

[1] Yuefei Bai, Qingsong Gao, Wei Jin: Modern Manufacturing Technology and Equipment. Vol.6(2009), p.29.

[2] Rongdi Kang, Feixue Yan. Ships Chemical Defense. Vol.6(2008), p.119.

[3] Zuoshi Liu, Xiaojuan Ni. Machine Mnufacturing.Vol.8(2004),p.32.

[4] Peng Liu,Qiuhong Kang. Journal of Chongqing Technology and Business University.Vol.6(2008) p.243.

[5] Yongqing Wu, Shuqui Liu, Guoxiong Zhang, Precision Engineering, Vol.28(2004),p89.

[6] Antonio Pirateli-Filho, Benedito Di Giacomo, Precision Engineering, Vol.27(2003),p283. 